\title{
Nucleating cavitation from laser-illuminated nano-particles
}

\author{
Caleb H. Farny, ${ }^{\text {a) }}$ Tianming Wu, R. Glynn Holt, Todd W. Murray, and Ronald A. Roy \\ Department of Aerospace and Mechanical Engineering, Boston University, 110 Cummington Street, Boston, \\ Massachusetts 02215
}

\begin{abstract}
Vapor bubble generation from laser-illuminated gold nanoparticles has been investigated as a means of providing nucleation sites for cavitation induced by high-intensity focused ultrasound (HIFU). Pulses from a 532-nm Nd:Yag laser were synchronized with a pulsed 1.1-MHz HIFU source in an acrylamide phantom seeded with 82-nm-diameter gold particles. Emissions from bubble collapses were detected by a $15-\mathrm{MHz}$ focused transducer at a laser pulse energy and HIFU focal pressure of $0.10 \mathrm{~mJ}$ and 0.92 $\mathrm{MPa}$, respectively. In comparison, a HIFU peak focal pressure of $4.50 \mathrm{MPa}$ was required to nucleate detectable cavitation without laser illumination.

(C) 2005 Acoustical Society of America

PACS numbers: 43.35.Ei, 43.80.Gx, 43.80.Sh

Date Received: March 1, 2005 Date Accepted: April 5, 2005
\end{abstract}

\section{Introduction}

The use of inertial cavitation as a mechanism for enhanced heating from high-intensity focused ultrasound (HIFU) has been an area of interest in therapeutic ultrasound applications. Cavitation has been shown to yield elevated heating rates over and above those produced by classical absorption in tissue ${ }^{1,2}$ and may provide a means for improving the efficacy of HIFU treatments. However, it is generally believed that pre-existing nucleation sites for cavitation are not omnipresent in most tissues in vivo. Ultrasound contrast agents (UCA) have been studied as a way of delivering nuclei into the target region, ${ }^{3-6}$ but UCA cavitation nuclei possess short lifespans due to diffusion and HIFU-induced destruction.

An alternative to UCA-based nucleation may be found by using minute optical absorbers in conjunction with laser irradiation. Laser-induced cavitation in water has been widely studied, ${ }^{7,8}$ and the growing area of optoacoustic imaging has shown that pulsed laser light can be used to produce cavitation from collapsing vapor bubbles surrounding gold nanoparticles conjugated to antibodies bound to the surface of breast cancer cells ${ }^{9}$ implanted at 5-6$\mathrm{cm}$ depths in vitro. Absorption of the laser energy by the particle causes rapid heating, leading to vaporization of the surrounding medium and the formation of a transient vapor cavity. If this cavity is larger than the Blake radius, subresonant in size [see Sec. 4.3.1 in Ref. 10] and formed during the rarefaction phase of the HIFU exposure, cavitation ensues. The size of the cavity is determined primarily by the size of the particle, and the arrival of the laser pulse determines the timing of nucleation relative to the acoustic cycle. With this technique, it may be possible to generate optimally sized nuclei within optically accessible regions, such as near the skin surface and in tissues possessing low optical absorption (such as breast). Such nuclei can be created essentially "on demand" and with minimal collateral damage as the absorption coefficient of the particles is much greater than that of the surrounding tissue.

Light absorption in gold nano-particles less than $100 \mathrm{~nm}$ peaks around $520 \mathrm{~nm} .{ }^{11} \mathrm{We}$ hypothesize that exposing a tissue-mimicking material seeded with gold nano-particles to 532 nm pulsed laser light possessing sufficiently high energy will produce transient vapor cavities ${ }^{12}$ that can serve as nucleation sites for HIFU-induced cavitation, effectively lowering the inertial cavitation threshold for the system. Here, we investigate this concept using 82-nm-diameter gold particles embedded in a transparent polyacrylamide gel. Cavitation activity was measured

${ }^{a}$ Electronic mail: cfarny@bu.edu 
$1 \mathrm{MHz}, 200$ cycles, $0.1 \mathrm{~Hz}$ prf $1.1 \mathrm{MHz}, 10$ cycles

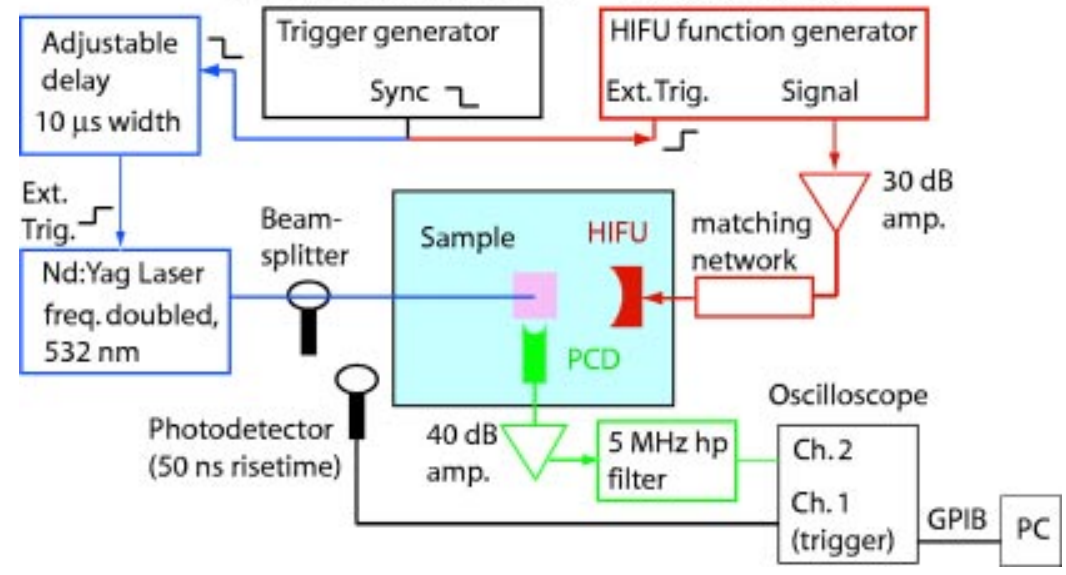

Fig. 1. Experimental arrangement.

as a function of HIFU pressure and laser energy, and the role of the timing of the laser pulse relative to the phase of the HIFU pressure field was investigated.

\section{Materials and methods}

All of the phantoms measured in this study consisted of a transparent acrylamide gel ( $7 \%$ concentration by volume, prepared in accordance with a procedure described in Ref. 13). This nondegassed material was "seeded" with 82 $\pm 6-\mathrm{nm}$ diameter gold particles (EM.GC80, BB International) at a concentration of $10^{9}$ particles $/ \mathrm{ml}$. The particles provide a pinkish hue but do not cloud the phantom and were more or less uniformly distributed in the gel.

A schematic of the experimental setup is shown in Fig. 1. The phantom was illuminated by a Q-switched, frequency-doubled Nd:Yag laser of 532-nm wavelength in TEM-00 mode with a 5-ns pulse length and 1.6-mm beamwidth (Minilite-10, Continuum). The pulse energy was controlled by a variable attenuator and was measured off-line with a power meter (MellesGriot). A 1.1-MHz transducer (H102-6, Sonic Concepts) was used as the HIFU source and was aligned coaxially to the laser beam via a two-axis positioning stage. A $15-\mathrm{MHz}$ focused broadband transducer (V313, Panametrics-NDT Inc.) was used in receive mode as a passive cavitation detector (PCD). The PCD was mounted on a three-axis positioning stage, oriented perpendicular and confocal to the HIFU beam axis. The phantom and acoustic transducers were submerged in distilled water in a glass-walled tank. The laser was operated externally to the tank.

Two function generators (33120A, Hewlett-Packard) and a custom-built gate and delay circuit were employed for the triggering of the laser and HIFU transducer. The laser was triggered by the sync signal from the "trigger generator" via an adjustable delay circuit. The sync signal from the trigger generator also triggered the "HIFU function generator," which output a 1.1-MHz ten-cycle sine burst to the HIFU transducer. The burst was amplified $30 \mathrm{~dB}$ before being sent to the transducer-matching network and HIFU transducer. The HIFU focal pressure was measured in water using a calibrated membrane hydrophone (Precision Acoustics, Inc.). The corresponding focal pressure in the phantom followed from a numerical simulation of the nonlinear pressure field given the known acoustical properties of the gel material $(1534 \mathrm{~m} / \mathrm{s}$ and $0.01 \mathrm{~Np} / \mathrm{cm} @ 1 \mathrm{MHz}$ ). The trigger generator was operated in burst mode at a $0.1-\mathrm{Hz}$ pulse repetition frequency (PRF), which established the repetition rate for both the laser and HIFU exposure. A low PRF was selected so that microcavitation bubbles generated by an HIFU burst did not linger in the phantom and serve as nucleation sites for the next burst. The timing between the onset of laser-induced nucleation and the HIFU pressure wave was varied by adjusting the 
laser trigger delay. This relative timing was established by measuring the light flash with an optical detector (PDA55, ThorLabs) and using a needle hydrophone (NP-4, DAPCO) to measure the acoustic transit time to the HIFU focus. The hydrophone was removed during the experiments in order to avoid cavitation on the surface of the needle.

The PCD signal from the 15-MHz receiving transducer was amplified $40 \mathrm{~dB}$ and highpass filtered above $5 \mathrm{MHz}$ (passive 4-stage Butterworth, Allen Avionics). The idea is that transient collapses from inertial cavities generate broadband acoustic emissions that superimpose on the primary HIFU field. Tuning the detector to pass high frequencies filters out acoustic energy at the fundamental HIFU frequency and achieves greater sensitivity to those emissions unique to inertial cavitation. The signal was digitized at $50 \mathrm{MHz}$ and displayed on an oscilloscope (Infinium, Hewlett-Packard) triggered by an optical detector adjacent to the laser beam path. PCD time-domain signals captured by the oscilloscope were downloaded via GPIB to a personal computer. The digitized real-time PCD signals included the electronic noise from the laser, background acoustic noise, and the broadband acoustic emissions generated by cavitation in the HIFU/PCD confocal zone. The data were analyzed by first applying a 5- $\mu \mathrm{s}$ rectangular window to the region of the signal which corresponded to the arrival time of the cavitation signals, thereby gating out noise from the laser firing. The emission level EL was then computed by taking the FFT of the time-gated signal and integrating the magnitude squared from $5 \mathrm{MHz}$ to half the sampling frequency; the EL was a measure of the broadband noise power emanating from the confocal region. Since inertial cavitation is the only significant source of broadband noise in the experiment (aside from the laser firing transient, which is gated out), the EL is thus a loose measure of inertial cavitation activity. The no-cavitation "background" signal was measured by blocking the laser beam and capturing the PCD signal when the HIFU and laser were firing. From this, the cavitation emission gain EG is defined as the EL, averaged over ten observations, expressed in $\mathrm{dB}$ relative to the background. The EG provides a measure of the level of cavitation activity over background occurring in the HIFU/PCD confocal region.

\section{Results}

We detected cavitation activity from the laser alone (no HIFU) and from the HIFU alone (no laser light). The minimum laser energy required to form PCD-detectable microcavitation was 4 $\mathrm{mJ}$. The minimum HIFU peak negative pressure required to produce detectable microcavitation was $4.50 \mathrm{MPa}$ and was the inertial cavitation threshold for the phantom used here.

The combined effect of the laser and HIFU was investigated by firing a $0.10-\mathrm{mJ} / \mathrm{pulse}$ and incrementing the HIFU pressure, pulse-to-pulse, until a cavitation signal was detected. Typical ungated PCD signals in the time- and frequency domains for two HIFU pressures are shown in Fig. 2. At $0.74 \mathrm{MPa}$ no cavitation signal was detected by the PCD; the signal at $0 \mathrm{~s}$ is noise generated by the laser firing. Cavitation acoustic emissions were clearly detected when the HIFU pressure was increased to $1.21 \mathrm{MPa}$, as indicated by the transient, high-amplitude signals in the time domain, and corresponded to an increase in the broadband emissions. The laser was fired when the third cycle in the ten-cycle HIFU burst arrived at the focus, and seven cavitation noise bursts were detected. The $12-\mu$ s arrival time of the cavitation signals corresponds to the acoustic transit time from the confocal region to the PCD.

In order to determine the role of the laser-HIFU timing on the cavitation activity, we initially set the laser to fire when the fifth HIFU cycle arrived at the focus; the pressure at this instant is zero and negative-going. The laser trigger delay was further incremented in steps of approximately $0.05 \mu \mathrm{s}$ for a full acoustic cycle, with ten measurements at each time delay, changing the arrival of the laser pulse incident to the HIFU cycle at the focus. The HIFU focal pressure was fixed at $1.25 \mathrm{MPa}$, and the laser energy was $0.12 \mathrm{~mJ} /$ pulse. The measured EG is plotted in Fig. 3 as a function of the relative phase between the laser firing and the HIFU field at the center of the confocal region. As the pressure approached peak rarefaction the cavitation emissions increased, and the signals decreased during the positive pressure phase. From this result a delay for an "optimum" phase for maximum cavitation activity was determined and was used to synchronize the HIFU and laser pulses.

Next, the HIFU cavitation threshold at the optimum phase was measured. Laser energy 

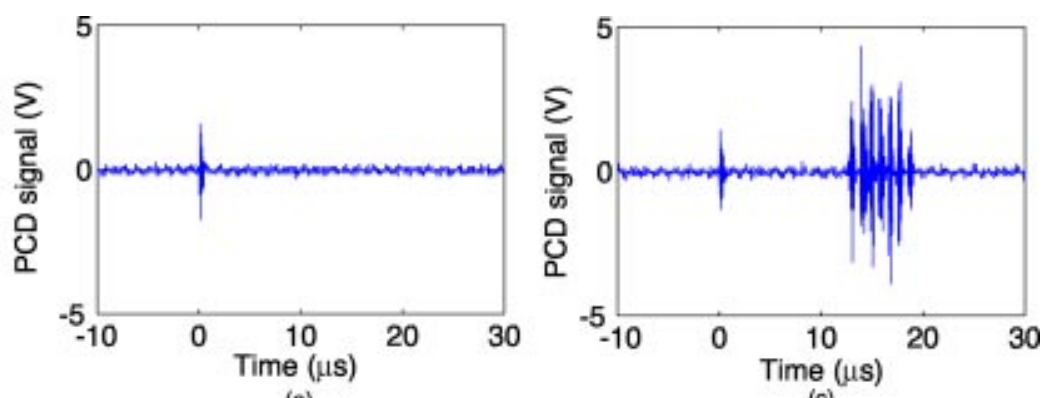

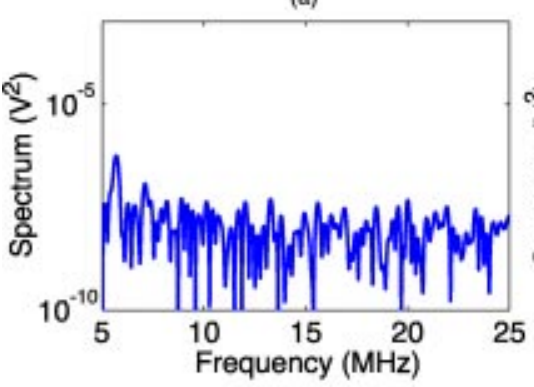

(b)

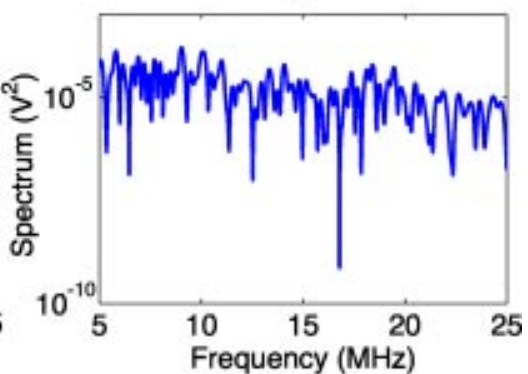

(d)

Fig. 2. PCD signals obtained with both the laser and HIFU on. The laser energy was $0.10 \mathrm{~mJ} /$ pulse. No cavitation signal was detected at a peak-negative HIFU pressure of $0.74 \mathrm{MPa}$ (a), (b). At 1.21 MPa a cavitation signal is evident in both the time-(c) and frequency-(d) domain plots.

settings of $0.07,0.09,0.10$, and $0.11 \mathrm{~mJ} /$ pulse were used; for each setting the HIFU pressure was increased by $0.09-\mathrm{MPa}$ increments from 0.8 to $1.4 \mathrm{MPa}$. The laser was timed to fire $0.15 \mu \mathrm{s}$ after the start of the fifth HIFU cycle arrived at the focus. Ten measurements for each combination were recorded and the average EG was calculated. The EG is plotted against the focal pressure in Fig. 4. Here, we can see a clearly demarcated threshold pressure (either 0.9 or $1.0 \mathrm{MPa}$, depending on the laser energy), with a gradual increase in the cavitation activity as the peak pressure was further increased. The cavitation threshold decreased as the laser energy was increased. No cavitation was detected at $0.07 \mathrm{~mJ} /$ pulse for any of the HIFU pressures employed.

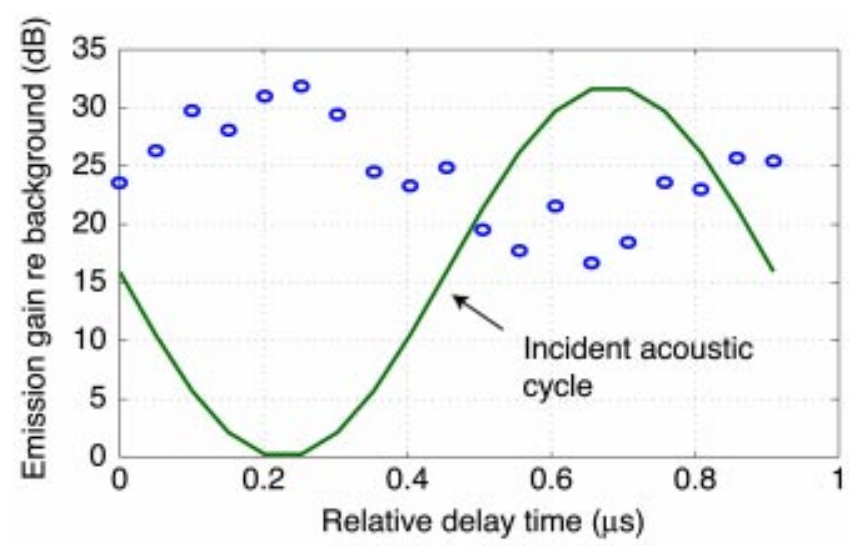

Fig. 3. The emission gain (EG) over the background signal relative to the timing of the laser pulse incident to the HIFU cycle. The laser energy was $0.12 \mathrm{~mJ} /$ pulse and the peak-negative HIFU pressure was $1.25 \mathrm{MPa}$. 


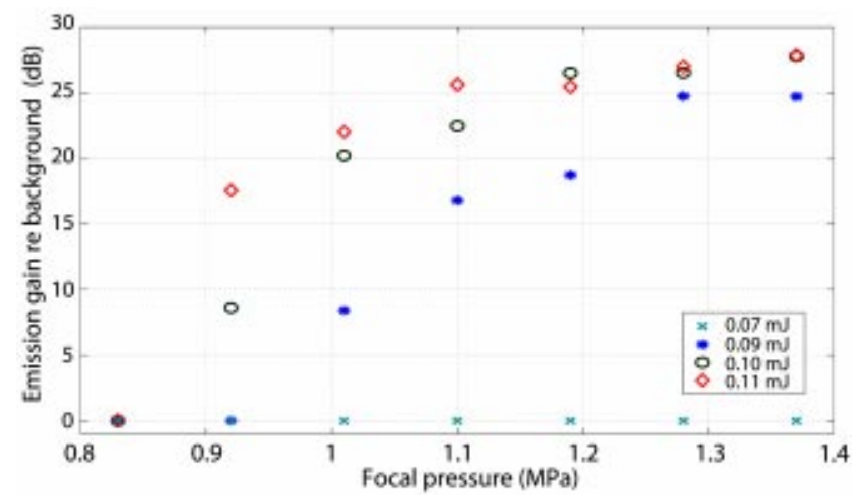

Fig. 4. The EG relative to the background as a function of HIFU peak-negative pressure and laser energy. The laser was fired $0.15 \mu \mathrm{s}$ after the fifth HIFU cycle arrived at the focus.

\section{Discussion and conclusions}

Laser illumination on the gold nano-particles proved effective in significantly lowering the HIFU cavitation threshold pressure. Cavitation was detected at peak-negative pressures as low as $0.92 \mathrm{MPa}$ when the laser pulse was synchronized with the HIFU signal, as compared to 4.50 $\mathrm{MPa}$ with no laser irradiation. There was no detectable reduction in the cavitation threshold pressure with the laser set to fire at $0.07 \mathrm{~mJ} /$ pulse; the threshold optical energy for generating a vapor cavity sufficient for cavitation nucleation appears to lie between 0.07 and $0.09 \mathrm{~mJ} / \mathrm{pulse}$ for the laser wavelength and particle sizes employed. Above this critical energy, increasing the laser energy served to lower the cavitation threshold and increased the amount of cavitation activity at a given pressure amplitude - at least up to about $0.12 \mathrm{~mJ} /$ pulse. The improved efficacy of HIFU cavitation nucleation is attributed to vapor-filled nano-bubbles produced from laser-heated gold nano-particles.

Measurements revealed that the timing of the laser pulse relative to the acoustic cycle has a large effect on the resulting cavitation activity. Signals were highest when the vapor bubble was produced during the peak rarefraction pressure phase, as one would expect. However, cavitation signals were still present when the laser was incident during the compression phase, an unexpected result. This is due to the fact that the optical and HIFU beams are collinear; thus, particles are being heated all along the spatial path of the HIFU pulse. There was always a location in which nuclei were generated in regions under acoustic stress, albeit at a somewhat lower peak negative pressure. This unfortunate ambiguity will be corrected in future work.

The reduction in threshold pressure with increasing laser energy is due to the increase in the size of the vapor nucleus. An energy-balance calculation yields an order-of-magnitude estimation of this size. The total energy absorbed by the particle in a single laser pulse $Q$ is given by $Q=\beta E_{0}\left(R_{S} / R_{L}\right)^{2}$, where $R_{S}$ is the particle radius, $R_{L}$ is the laser beam radius $(0.8 \mathrm{~mm})$, and $E_{0}$ is the laser pulse energy $(0.1 \mathrm{~mJ})$. From Mie theory ${ }^{11}$ the absorption efficiency $\beta$ is $3.3(40 \mathrm{~nm}$ radius gold, $532 \mathrm{~nm}$ laser), so $Q$ is $0.87 \mathrm{pJ}$. Modeling the interface as a thin liquid at $300 \mathrm{~K}$ and $0.1 \mathrm{MPa}$, the interface has a pressure jump due to the surface tension when evaporation starts. The Laplace pressure in water can be approximated by $2 \sigma / R_{s}=3.6 \mathrm{MPa}$, which corresponds to $520 \mathrm{~K}$ for the saturation temperature. Assuming all the energy absorbed by the particle is consumed in heating and evaporation processes, a $14.3-\mathrm{nm}$ shell around the particle is instantaneously vaporized. Subsequent expansion of this volume results in a cavity with a radius of approximately $105 \mathrm{~nm}$. Gas diffusion into this vapor cavity (not modeled) will cause it to be somewhat larger and could promote a rebound upon collapse that will help to sustain bubble activity from one acoustic cycle to the next. The repeated period emissions shown in Fig. 2(c) serve as evidence of this phenomenon.

It is instructive to consider how this estimate compares with expectations based on the 
measured cavitation threshold pressures. At $\mathrm{MHz}$ frequencies, the optimum nuclei size for inertial cavitation is somewhat larger than the Blake radius, ${ }^{14}$ which decreased with increasing acoustic pressure amplitude. ${ }^{10}$ The lowest "laser-nucleated" threshold pressures measured in this study suggest a corresponding Blake radius of about $70 \mathrm{~nm}$; this corresponds to the largest value of the Blake radius encountered in these experiments. The difference between the computed laser-induced cavity size and the Blake radius may not be significant, particularly given the simplicity of the model employed. Nevertheless, the fact that the former is greater than the latter is consistent with physical expectations.

\section{Acknowledgments}

The authors gratefully acknowledge the financial support of the Center for Subsurface Sensing and Imaging Systems via NSF ERC Award Number EEC-9986821, and the technical assistance of Mr. Gopi Maguluri.

\section{References and links}

${ }^{1}$ R. G. Holt and R. A. Roy, Ultrasound Med. Biol. 27, 1399-1412 (2001).

${ }^{2}$ K. Hynynen, Ultrasound Med. Biol. 17, 157-169 (1991).

${ }^{3}$ D. L. Miller and R. M. Thomas, Ultrasound Med. Biol. 21, 1059-1065 (1995).

${ }_{5}^{4}$ D. L. Miller and R. A. Gies, Ultrasound Med. Biol. 27, 1201-1208 (1988).

${ }^{5}$ E. Sassaroli and K. Hynynen, J. Acoust. Soc. Am. 115, 3225-3243 (2004).

${ }^{6}$ B. C. Tran, J. Seop, T. L. Hall, J. B. Fowlkes, and C. A. Cain, IEEE Trans. Ultrason. Ferroelectr. Freq. Control 50, 1296-1304 (2003).

${ }^{7}$ M. P. Felix and A. T. Ellis, Appl. Phys. Lett. 19, 484-486 (1971).

${ }^{8}$ W. Lauterborn and H. Bolle, J. Fluid Mech. 72, 391-399 (1975).

${ }^{9}$ M. Eghtedari, M. Motamedi, V. L. Popov, N. A. Kotov, and A. A. Oraevsky, Photons Plus Ultrasound: Imaging and Sensing, Proceedings of SPIE, edited by A. A. Oraevsky and L. V. Wang (SPIE, Bellingham, WA, 2004), Vol. 5320, pp. 365-377.

${ }^{10}$ T. G. Leighton, The Acoustic Bubble (Academic, London, 1997).

${ }^{11}$ H. C. van de Hulst, Light Scattering by Small Particles, 1st ed. (Dover, New York, 1981).

${ }^{12}$ V. Zharov and D. Lapotko, Rev. Sci. Instrum. 74, 785-788 (2003).

${ }^{13} \mathrm{X}$. Yang, "Investigation of bubble dynamics and heating during focused ultrasound insonation in tissuemimicking materials," Ph.D. thesis, Boston University (2003).

${ }^{14}$ R. E. Apfel and C. K. Holland, Ultrasound Med. Biol. 17, 179-185 (1991). 